Marek Jakubiec

\title{
Extrapolative Inference and Analogical Reasoning in the Empirical Sciences
}

In the present paper, I am going to propose a distinction between two types of inferences which are used in the empirical sciences: extrapolation and per analogiam reasoning. First, this issue is interesting due to the practical relevance of both of them and the necessity of that distinction, which is of great importance from the methodological and philosophical perspectives. Second, there are not many publications dedicated to its deeper analysis from the perspectives of analytical philosophy and the philosophy of science.

Several publications ${ }^{1}$ that have appeared in recent years have undoubtedly made an important contribution to helping fill this gap, but the sensu stricto philosophical analysis is still at an early stage. Obviously, my aim in this text is not to fill this void; rather, my goal is to identify some important issues that are necessary to answer the question of the nature and significance of extrapolation in the empirical sciences.

In addition to an analogy, there is also a need to consider differences that exist between extrapolation and induction as well

1 See, for instance: D. Steel, Across the Boundaries. Extrapolation in Biology and Social Science, Oxford 2008; F. Guala, Extrapolation, Analogy and Comparative Process Tracing, „Philosophy of Science” 2010, Vol. 77, pp. 1070-1082; M. Heller, Zasady ekstrapolacji. Uwagi na marginesie kosmologii, „Zagadnienia Filozoficzne w Nauce” 1978/79, nr 1, pp. 23-31. 
as statistical inference. This text should therefore be regarded as a brief introduction to this topic, as I will limit myself to indicating the most important differences between the proposed definition of extrapolation and reasoning by analogy, which also plays an impossible to ignore role in the formulation of scientific claims.

In light of these comments, some theses presented in the paper may be controversial. I will be very grateful for any constructive criticism and suggestions.

\section{What is extrapolative inference?}

It is not easy to briefly describe the essence of extrapolative inference in a way that does not miss any of the extremely important issues associated with it, especially considering the relatively small amount of literature concerning this issue from the philosophical perspective. Even then I will make an attempt at conducting a synthetic analysis of issues relevant to us, paying attention to some of those that are the most interesting from the point of view of the explication of the relationship between per analogiam reasoning and extrapolation.

I limit these considerations to extrapolation inference in the empirical sciences. Hence it is necessary to start by pointing to an obvious, but in this context also quite important assertion. Therefore, several features of scientific sentences are significant from the point of view of the analysis of the epistemic character.

First, these are generality and universality; namely, the truthfulness in the sense similar to the classic meaning.

However, the existence of both the first and second of these characteristics is questionable in light of the cognitive limitations of the human intellect, in particular its temporal and spatial dimensions.

Therefore, it is noticeable that there is a certain tension between, on the one hand, actual cognitive capabilities that affect the ability to recognize certain statements as legitimate and credible and, on the other, the sentences formulated by representatives of the natural sciences that describe the phenomena occurring in the world, 
i.e., the laws of nature. I will try to clarify that issue in my further remarks, but now I will explain the perspective they are turned into.

It should be stressed that these considerations are carried out from the perspective of scientific realism, the view according to which science generally describes existing reality, and the belief in this description is justified. It is relevant to indicate that presuppositions due to the acceptance of an anti-realistic attitude undermine the sense of considering the problem of the legitimacy of both the scientific statements and nature of the aforementioned tension. Of course, anti-realism is not a homogenous position, and analyzing its varieties is far beyond the interest of this paper. Therefore, I will confine myself to two of its types.

First, following the trend of conceptual anti-realism (which is, in fact, a form of epistemological relativism) we recognize that it is not possible to access the "objective" reality, and our knowledge may be built only by more or less perfect conceptual schemes. The issue of the relationship between universal sentences and possessed "knowledge" becomes less important, as scientific ascertainments in the context of such a position do not fully and accurately describe the objective existing reality, and thus do not have the status of universality. The acceptance of a simpler version of anti-realism, according to which it is not possible to know actual reality (this is in many aspects identical to skepticism) causes the extrapolation problem to become devoid of any significance; it cannot be an important epistemological issue any longer, for if we learn that the acquisition any kind of knowledge is not possible, neither is it possible to infer by extrapolation.

Consequently, as stated previously, there is a certain tension and perhaps even a paradox associated with the nature of our scientific cognition in the realistic perspective. Above all, our knowledge is always fragmentary due to the limited capacity of our intellect. Our aim, however, is to formulate general statements, which articulate universal (which, therefore, are in force across the entire universe at any time) laws of nature in a manner consistent with the real state of things (and thus sentences are true in the classical sense). Despite these limitations, what is crucial is that science 
"works": we can make predictions, and it is possible to use acquired knowledge for specific purposes (one can call them "technology"). This fact leads to the conclusion that the goal to which we aspire, as mentioned above, is at least partially achieved.

At this point an extremely important question from the philosophical point of view comes to light: how is it possible that science "works", although it is impossible to formulate peremptorily true sentences?

To take part in the discussion on this issue, it is necessary to indicate the nature of this impossibility.

First, it is not possible to clearly determine the veracity of a particular general statement describing a state of affairs or, in other words, its verification. This would require an analysis of all possible cases described by the sentence.

In view of the limitations of human cognition, this appears as impossible neither in theory nor in practice. The sentence is true not only at one moment (hic et nunc), but it is so always. As a result, in order to legitimately declare its truthfulness, it would be necessary to revise the sentence in relation to the states of affairs that occurred in the past and those that will take place in the future.

This causes verification to be impossible even in theory: a finite number of available observations will never be enough. As Rudolf Carnap has noted:

If verification is understood as a complete and definitive establishment of truth, then a universal sentence, such as a so-called law of physics or biology, can never be verified, a fact which has often been remarked. Even if each single instance of the law were supposed to be verifiable, the number of instances to which the law refers - i.e., the space-time-points - is infinite and therefore can never be exhausted by our observations which are always finite in number. ${ }^{2}$

2 R. Carnap, Testability and Meaning, [in:] Readings in the Philosophy of Science, ed. H. Fleigl, M. Brodbeck, New York 1999, p. 48. 
Moreover, even the particular sentence concerning the one certain fact is not fully verifiable, because it is always possible to contest the results of observation or to request the verification of the results, check the checking, etc.

In spite of the fact that we are not able to verify any general sentence describing reality, we can test arising "particular sentences that we derive from the law and from other sentences established previously."3

If we do not encounter any case against it, namely falsifying (or rather, in the context of Carnap's philosophy, disconfirming) a particular sentence, the general law of nature becomes somehow more "reliable". According to Carnap, this procedure "gradually [increases] confirmation of the law."4

An attempt to solve the problem by introducing the concept of falsification was proposed by Karl Popper. He recognized that while it does not allow us to prove a theory, logic allows for the experimental overthrow or falsification of a theory, which is in accordance with the modus tollendo tollens. ${ }^{5}$ To put it simply, hypothesising and testing will provide us asymptotic approximation to the truth. Thus it is wrong to believe that our theories are either true or false. They will most likely all be false, but not all of them already will have been refuted by that time. ${ }^{6}$ At most, as Popper notes, corroboration ${ }^{7}$, or recognition that the theory "has proved hart" is possible. This, of course, also cannot be considered to be a sufficient criterion for the recognition of the full adequacy or genuineness of a particular hypothesis. Moreover, the finding that a falsification can clearly reject a statement as false is also not undisputed.

As "pure" facts do not exist and each observation is based on a theoretical component, statements cannot be fully falsified (see

\footnotetext{
3 Ibid.

4 Ibid.

5 K. Popper, Logic of Scientific Discovery, London-New York 2005, pp. $18 \mathrm{f}$.

6 W. Sady, Falsyfikacjonizm Karla Poppera, [in:] W. Sady, Spór o racjonalność naukowa. Od Poincarego do Laudana, Wrocław 2000.

7 K. Popper, Logic..., pp. 248 f.
} 
Duhem-Quine thesis, according to which testing in science cannot be described by modus tollens because no theory is ever examined in isolation, but together with some other beliefs, and therefore in the case of a negative result of experiment it is not known whether the theory or background knowledge is refuted). Moreover, falsification is always a greater or lesser degree of coincidence. It is possible that such circumstances could prevent it in spite of taking efforts to occur in this direction.

The (of course, very briefly) presented attempts to solve the problem I call ad hoc that of the truth of the statements of science derive from the conclusion that it is not possible to establish the truth of general statements. Therefore, we should consider whether it is reasonable to say that the most important aspect of the scientific method is to infer by extrapolation of the results of only de facto confirmed statements (or theses which "proved hart").

The crucial importance of this question may be noticed in the context of cosmology (but also physics in general) because, on the one hand we possess the knowledge (i.e., set of sentences that we consider reliable) regarding only a (relatively) small area of the universe and, on the other hand, we formulate general laws which are in force in the whole universe. This way we extrapolate (we infer by extrapolation) the known results, but only on this stretch of reality, to the whole, de facto unknown reality.

Is such a procedure justified? Answering this question is not effortless. Most important, we have to notice that it is not identical with induction, although one can find some similarities. Both of them are, as I will later point out, types of reasoning by generalization.

As I mentioned above, the concept of "extrapolation" is in fact treated primarily as a mathematical problem in the literature. However, even definitions that can be found in statistics textbooks may be useful for our philosophical analysis. For instance, its statistical definition is:

Extrapolation is based on the estimation of a hypothetical value beyond the study period. Statistical estimation is used in cases in which it is impossible or pointless to make use of a full or even a partial study. 
It also points to the essence of extrapolation within the meaning of the philosophy of science.

I propose the following philosophical definition of extrapolative inference:

The formulation of general theorems concerning all objects and states of affairs (laws of nature in the context of the empirical sciences) on the grounds of knowledge (with the objections made above), only a part of them concerning certain aspects of the whole.

This is similar to the aforementioned mathematical extrapolation definition relating to the function, according to which it is an

[e]xtention (a graph, curve or range of values) by inferring unknown values from trends in the known data. ${ }^{8}$

This also shows the substance of extrapolation within the meaning of philosophy.

In connection with the presentation of the extrapolation issue it becomes necessary to analyze the differences between it and the terms whose meaning is similar, which may be identified with induction, analogy and statistical inference. In the following sections of this article I will address the relationship between extrapolative inference and analogical reasoning.

\section{Briefly about analogies in science}

The differences existing between extrapolative inference and analogy are significant. However, they may remain unnoticed, mainly due to the poor development of the philosophical approach to the problem of extrapolation. Therefore, I will begin by pointing out the most important and most frequently cited characteristics of per analogiam reasoning. Then I will show the key differences between analogy and extrapolation used in the natural sciences. Al-

8 Extrapolate, www.oxforddictionaries.com/definition/english/extrapolate (20.08.2014). 
ready at this point it should be noted, however, that analogy plays a significant role ${ }^{9}$, especially in the case of special issues, which eventually proves extrapolative inference to be the most useful.

It is difficult to concisely describe the essence of analogical reasoning due to its multifaceted and significant differences in its interpretation; therefore, I will confine it to the adoption of its specific understanding.

I will begin with a simple example. I assume that there are two states of affairs (this term will collectively determine the investigated objects, events, etc.): $A$ and $A$; in which $A$ is the Earth, and $A$ ' is a planet discovered hundreds of light years from our solar system, similar to Earth (referring to the nomenclature of Putnam's famous example, I will call her "Twin Earth").

The resemblance between the previously studied object and the one that is the target of the study is the first requirement of analogy, which is also a conditio sine qua non for believing it to be a cognitively valuable procedure. Mary Hesse calls it a material analogy and considers it to be a necessary element of any acceptable analogical argument. That similarity should imply the existence of some observable (or "pre-theoretic") properties. ${ }^{10}$

Furthermore, the state of affairs in $A$ has been relatively well-studied, the theory regarding $A$ was empirically tested, all the predictions were confirmed and the researchers did not encounter any contradictory event that would falsyfy the theory. Our theory of the state of $A$ may therefore be regarded as credible, and "corroborated”, using Popper's concept. Speaking in colloquial language, we may conclude that we have knowledge about it.

At a certain moment it turns out that there is a detached state of affairs in $A$ ' for which we can say, due to some circumstances known to us, that it is probably relevantly similar to the state of $A$. For whatever reason we are not able to perform the same tests

9 See for instance: A. Biela, 1991, [in:] A. Biela, Analogy in Science. From a psychological perspective, Frankfurt-New York 1991; M. Hesse, The Structure of Scientific Inference, Berkeley 1974.

10 M. Hesse, Models and Analogies in Science, Notre Dame 1966, p. 68 f. 
as in a case of the state of affairs of $A$ with respect to $A^{\prime}$. Therefore, on the basis of the idea of this similarity, we recognize that the description of the state of $A$ is also likely to be adequate to describe the state of $A^{\prime}$, and then perform the description of $A^{\prime}$.

This discussed simple example shows that the analogy is a type of reasoning used to determine the characteristics, values, etc. of certain objects, which for various reasons are impossible to directly examine. A limited description of them lets us to assume that they exhibit significant similarity (at least in some aspects) to the objects that we can adequately describe. Therefore, on the base of the assumption concerning similarity, we formulate hypotheses about unknown objects.

They currently provide the source of knowledge about them that is most adequate to the highest possible degree. The results gained through the application of per analogiam reasoning cannot, however, be regarded as the fully correct description of $A^{\prime}$ since they are merely a sort of approximation, based additionally on the always more or less questionable assumption of similarity of the two states of affairs.

Two features seem to be decisive and picture the essence of the analogical reasoning used in science. First, it always refers to the specific states of affairs and, secondly, is based on the explicit assumption that concerns the similarity of equal states of affairs. Finally, obtained via analogy results are, ex definitione, devoid of the claim to authenticity. These issues present themselves quite differently if considered in light of extrapolation and it causes these two tools to vary significantly.

\section{Analogy and extrapolation}

First of all, extrapolation does not concern the specific (concrete) cases, but the general sentences. These cases therefore relate only indirectly. The essence of extrapolation in the proposed definition is not limited to describing the unknown states of affairs on the base of knowledge pertaining to the known, but to formulate on the basis of a limited amount of acquired data and theoretical 
apparatus sentences, which pretend to be universally true. Even though it may be useful in the formulation of hypotheses, analogy cannot be regarded as a decisive element of the scientific method.

Secondly, the extrapolative inference does not contain an explicite expressed limitation, unlike in the case of the analogy, whose explanatory efficacy is strongly limited by reception at the starting point in the thesis of similarity, which in itself is merely a sort of working assumption. Laws of nature formulated by extrapolation are unconditional and, from the perspective of scientific realism, claim full "veracity", i.e., they describe the phenomena in accordance with the real states of affairs.

However, it is also worth considering whether the analogy is not some kind of extrapolation in the narrower sense. Similarly as the latter, it also relies on a kind of generalization. The description of the known state of affairs (referring to our example $A$ ) is, in fact, generalized in the sense that it turns out to be useful under certain conditions also for the formulation of descriptions of the state of $A$ : The differences between the two tools, however, indicate that there is a need to distinguish them to prevent possible confusion. At most, we can talk about the existence of a wider group of reasoning by generalization (although this term is not fully adequate for the analogy).

\section{Resume}

The first aim of this paper was to present the issue of extrapolation, which seems to be very interesting to study from the perspective of contemporary philosophy of science. For its proper explanation, it is crucial to distinguish this term from other concepts of similar meaning, such as analogy, induction or statistical inference. The second goal is to indicate key differences that exist between extrapolative inference and analogical reasoning. This allows for the ascertaining of the identification of these concepts, which would be a mistake. However, there is no way to not notice certain similarities.

Although the objectives of extrapolation and analogy in the presented meanings are diametrically opposed, there are certain simi- 
larities between these two procedures. In both cases (as well as in the induction and statistical inferences) there occurs something that can be described as the "expansion of knowledge" in areas of research we know less about or which remain fully puzzling. Only in this sense, then, it will be justified to determine analogy, induction and statistical inference as "extrapolative inferences". However, it would be better to refrain from this wording to avoid ambiguity.

\section{Summary}

The primary purpose of this paper is to present the issue of extrapolation, which is interesting from the perspective of contemporary philosophy of science. For its proper explanation, it is crucial to distinguish it from terms of similar meaning, such as analogy, induction or statistical inference. The second goal is to indicate key differences that exist between extrapolative inference and analogical reasoning. Because of this the ascertaining of the identification of these concepts would be a mistake though, of course, there is no way not to notice certain similarities.

Keywords: scientific method, extrapolation, per analogiam reasoning, philosophy of science, generalization

\section{Bibliography}

Biela A., Analogy in Science. From a psychological perspective, FrankfurtNew York 1991.

Carnap R., Testability and Meaning, [in:] Readings in the Philosophy of Science, ed. H. Fleigl, M. Brodbeck, New York 1999, p. 47-92.

Extrapolate, www.oxforddictionaries.com/definition/english/extrapolate (20.08.2014).

Guala F., Extrapolation, Analogy and Comparative Process Tracing, "Philosophy of Science" 2010, Vol. 77, pp. 1070-1082.

Hesse M., Models and Analogies in Science, Notre Dame 1966.

Hesse M., The Structure of Scientific Inference, Berkeley 1974.

Popper K., Logic of Scientific Discovery, London-New York 2005.

Sady W., Falsyfikacjonizm Karla Poppera, [in:] W. Sady, Spór o racjonalność naukowa. Od Poincarego do Laudana, Wrocław, 2000.

Steel D., Across the Boundaries. Extrapolation in Biology and Social Science, Oxford 2008. 